\title{
On bilevel machine scheduling problems
}

\author{
Tamás Kis (Speaker) * András Kovács ${ }^{\dagger}$
}

\section{Introduction}

Bilevel optimization is concerned with two-level optimization problems, where there is a top level decision maker or leader, and there is one (or more) bottom level decision maker or follower. Each decision maker optimizes its own objective function and is affected by the actions of the other. The follower makes its decisions after, and in view of, the decisions of the leader. For an overview and references, see [1].

There are only sporadic results for solving bilevel machine scheduling problems, see e.g., [2], [3]. However, such models may occur naturally, as we demonstrate it next. Suppose there is a shop with parallel machines and two decision makers: a top level planner who decides about the assignment of jobs to machines, and a bottom level shop manager who decides about the processing order of jobs on each machine. The production planner is concerned with customers, and aims at minimising the total weighted completion time with job weights $w_{j}^{1}$. In contrast, the shop manager is concerned with production costs and technology, which means that there is a most economical processing order on each machine. To impose such an ordering, the shop manager sets appropriate job weights $w_{j}^{2}$ and requires that the jobs assigned to each machine be processed in weighted-shortest-processing-time (WSPT) order. Since the WSPT order minimizes the weighted flow time, the shop manager can equivalently require that the schedule of each machine has to minimize the total weighted flow time with respect to jobweights $w_{j}^{2}$. Clearly, the planner aims to find an assignment of jobs to machines such that his objective function is minimized knowing the strategy of the shop manager. If he is optimistic, he may assume that the shop manager would cooperate, i.e., in case of ties with respect to weights $w_{j}^{2}$, the machines will process the jobs with larger $w_{j}^{1} / p_{j}$ ratio (WSPT order). However, if he was pessimistic, then he prepares for the worst case, i.e., in case of ties with respect to weights $w_{j}^{2}$, the machines will process the jobs with smaller $w_{j}^{1} / p_{j}$ ratio (anti WSPT order).

Notice that such a solution cannot be modelled by imposing precedence constraints among the jobs, because the assignment of jobs to machines is not known in advance.

In the following we formalize bilevel optimization problems and discuss some results on two basic machine scheduling problems: (1) bilevel scheduling of parallel machines with resource allocation at the top level and sequencing at the bottom level, and weighted completion time minimization at both levels, and (2) bilevel single machine scheduling with accept/reject decisions at the top level for minimising the weighted number of rejected jobs, and sequencing at the bottom level for minimising the weighted completion time.

*tamas.kis@sztaki.hu. Computer and Automation Institute, Kende str 13-17, 1111 Budapest, Hungary.

†akovacs@sztaki.hu. Computer and Automation Institute, Kende str 13-17, 1111 Budapest, Hungary. 


\section{Bilevel optimization}

There are two basic scenarios. In the optimistic case the leader trusts the follower that it will help him to achieve the best objective function, i.e., in case of ties, the follower chooses an optimal solution which is the most advantageous for the leader. Mathematically, assuming that both the leader and the follower minimize its objective function, the optimistic bilevel optimization problem can be formalized as follows:

$$
\begin{array}{r}
\min _{x, y} f(x, y) \\
\text { s.t. }(x, y) \in \Omega \\
y \in \arg \min _{w} g(x, w) \\
\text { s.t. }(x, w) \in \Psi .
\end{array}
$$

Here, the leader's objective function and set of feasible solutions are $f$ and $\Omega$, respectively, and $g$ and $\Psi$ are those of the follower. As can be seen, the follower decides only $y(w)$, and $x$ is a parameter, imposed by the leader. In contrast, in the pessimistic case, the leader aims at minimising the loss caused by the follower if he chooses the optimal solution which is the least advantageous for the leader:

$$
\begin{array}{r}
\min _{x, y} \max f(x, y) \\
\text { s.t. }(x, y) \in \Omega \\
y \in \arg \min _{w} g(x, w) \\
\text { s.t. }(x, w) \in \Psi .
\end{array}
$$

For more details, see [1].

\section{The bilevel weighted flow time problem}

In this problem, there are $n$ jobs and $m$ parallel identical machines. Each job has a processing time $p_{j}$ and two weights, $w_{j}^{1}$ and $w_{j}^{2}$. The leader assigns jobs to machines, and the follower orders the jobs assigned to each machine. The follower's objective is to minimize $\sum_{i=1}^{m} \sum_{j \in J_{i}} w_{j}^{2} C_{j}$, where $M_{i}$ is the set of those jobs assigned to machine $i$. In the optimistic case, the leader's objective is to minimize the weighted completion time $\sum_{j=1}^{n} w_{j}^{1} C_{j}$, whereas in the pessimistic case its objective is to minimize $\max \sum_{j=1}^{n} w_{j}^{1} C_{j}$. The leader aims to find the sets $A_{i}$ such that its decision is optimal in the optimistic or in the pessimistic sense.

We show that this problem is NP-hard in the strong sense in general. Moreover, both in the optimistic and in the pessimistic case there is a global ordering of jobs $j_{1} \prec j_{2} \prec \cdots j_{n}$, such that if the set of jobs assigned to machine $i$ is $J_{i}=\left\{\pi_{1}^{i}, \ldots, \pi_{n_{i}}^{i}\right\}$, then it is optimal for the follower to process the jobs on machine $i$ in $\prec$ order, i.e., job $\pi_{k}^{i}$ is processed before job $\pi_{\ell}^{i}$ on machine $i$, iff $\pi_{k}^{i} \prec \pi_{\ell}^{i}$. Moreover, this ordering gives the lowest objective function value for the follower with respect to any assignment of jobs to machines.

The above global order leads to a reformulation of the bilevel scheduling problem as a MAX $m$-CUT problem with special arc weights. We will also discuss a non-trivial polynomially solvable special case of the bilevel scheduling problem. As a by-product, the latter provides a new polynomially solvable case of the MAX $m$-CUT problem. 


\section{The bilevel order acceptance problem}

Suppose there is a production line and two decision makers: a planner (leader) who accepts or rejects the jobs, and a scheduler (follower) who decides about the processing sequence of accepted jobs. Each job has a processing time $p_{j}$, a deadline $d_{j}$, and two weights, $w_{j}^{1}$ and $w_{j}^{2}$. Accepted jobs have to be completed before their deadlines. The leader's objective is to maximize the total weight of accepted jobs, or alternatively, minimize the total weight of rejected jobs. In contrast, the follower solves the scheduling problem $1 \| \sum_{j} w_{j}^{2} C_{j}$. The leader has no influence on the sequence of selected jobs, and the follower does not take into consideration the deadlines. If the leader is optimistic, he has to select such a subset $A$ of jobs that admits an optimal sequence with respect to the follower's objective $\min \sum_{j \in A} w_{j}^{2} C_{j}$ such that $C_{j} \leq d_{j}$ for each job $j \in A$, and $\sum_{j \in A} w_{j}^{1}$ is maximal. If the leader is pessimistic, then he chooses such a subset $A^{\prime}$ of jobs such that every optimal solution of the scheduling problem $1 \| \sum_{j \in A^{\prime}} w_{j}^{2} C_{j}$ satisfies the condition $C_{j} \leq d_{j}$ for all $j \in A^{\prime}$. Note that the leader's objective depends only on his selection of jobs, but the feasibility of the selection depends on the follower's optimal sequence.

We show that the decision version of the order-acceptance problem is NP-hard and provide a dynamic program of pseudo-polynomial time complexity for solving it. For the special case with $w_{j}^{1} \equiv 1$, we adapt the Moore-Hodgson algorithm for $1 \| \sum_{j} u_{j}[4]$.

\section{Acknowledgements}

The work reported here has been supported by OTKA grant K76810, and András Kovács acknowledges the support of the Bolyai János research grant BO/00138/07.

\section{References}

[1] S. Dempe, Annotated bibliography on bilevel programming and mathematical programming with equilibrium constraints, Optimization 52 (2003) 333-359.

[2] J.K. Karlof and W. Wang, Bilevel programming applied to the flowshop scheduling problem, Computers and Operations Research 23 (1996) 443-451.

[3] Z. Lukač, K. S̆orić, and V.V. Rosenzweig, Production planning problem with sequence dependent setups as a bilevel programming problem, European Journal of Operational Research, 187 (2008) 1504-1512.

[4] J.M. Moore, An $n$ job, one machine sequencing algorithm for minimizing the number of late jobs. Management Science 15 (1968) 102-109. 